\title{
Evaluation of the Anti-inflammatory Effects of Atorvastatin on Patients with Rheumatoid Arthritis: A Randomized Clinical Trial
}

Zhaleh Shariati Sarabi ${ }^{1}$, Mehran Ghazi Saeidi ${ }^{2}$, Mandana Khodashahi ${ }^{3}$, Ali Etemad Rezaie ${ }^{4}$, Kamila Hashemzadeh $^{3}$, Rozita Khodashahi ${ }^{5}$, Hossein Heidari ${ }^{6}$

${ }^{1}$ Associate Professor, Rheumatology Department, Rheumatic Diseases Research Center, School of Medicine, Mashhad University of Medical Sciences, Mashhad, Iran

${ }^{2}$ Assistant Professor, Rheumatology Department, North Khorasan University of Medical Sciences, Bojnord, Iran

${ }^{3}$ Assistant Professor, Rheumatology Department, Rheumatic Diseases Research Center, School of Medicine, Mashhad University of Medical Sciences, Mashhad, Iran

${ }^{4}$ M.Sc., Chicago Medical School, Rosalind Franklin University, North Chicago, Illinois, USA

${ }^{5}$ Infectious Disease Resident, Department of Infectious Diseases. Imam Reza Hospital, Mashhad University of Medical Sciences, Mashhad, Iran

${ }^{6}$ Medical Student, Mashhad University of Medical Sciences, Mashhad, Iran

\section{Type of article: Original}

\begin{abstract}
Background: Rheumatoid arthritis (RA) is a chronic inflammatory joint disorder with unknown etiology. Atorvastatin is a lipid-lowering agent that affects the inflammatory processes.

Objective: This study aimed to determine the anti-inflammatory effects of atorvastatin on the Disease Activity Index and high-density lipoprotein (HDL) concentrations in RA patients.

Methods: This clinical trial was performed on 38 RA patients, who were referred to the Imam Reza and Ghaem Medical Centers of Mashhad, Iran between 2013 and 2014. Patients were divided into two groups: 1) the intervention group, which received $40 \mathrm{mg}$ of atorvastatin, and 2) the control group. Response to treatment and the clinical status of patients were evaluated using the Disease Activity Score (DAS-28) and Visual Analogue Scale (VAS) at weeks zero, four, eight, and twelve, based on the 2010 ACR/EULAR Criteria by two rheumatologists. Disease activity and laboratory parameters, including erythrocyte sedimentation rate (ESR), high-sensitivity Creactive protein (hs-CRP), DAS-ESR, DAS- hs-CRP, high-density lipoprotein (HDL), low-density lipoprotein (LDL), and liver function test (LFT) were measured in both groups.

Results: There was a significant difference in the mean number of swollen joints $(p<0.011)$, ESR $(p<0.005)$, DAS-ESR ( $<<0.043)$, LDL (0.036), and HDL (0.016) between the two groups. The changes in trend showed no significant difference in the mean number of tender joints $(p=0.38)$, VAS $(p=0.715)$, CRP $(p=0.07)$, DAS-hsCRP $(p=0.431)$, total cholesterol $(p=0.285)$, or TG $(p=0.331)$ between the two groups. However, the Disease Activity Index decreased by $48.4 \%$ in the intervention group, compared to $35.5 \%$ in the control group.

Conclusion: As the results indicated, atorvastatin has a positive effect on the course of RA. In fact, atorvastatin, as an anti-inflammatory agent, could significantly influence inflammation in RA patients. Therefore, adding a lipid-lowering agent to standard medications in RA may be warranted and could decrease disease activity.

Clinical trial registration: The trial was registered at the Iranian Registry of Clinical Trials (Website: http://www.irct.ir, Irct ID: IRCT2015122425648N2).

Funding: The authors received no financial support for the research, authorship, and/or publication of this article. Keywords: Atorvastatin; Rheumatoid arthritis; Anti-inflammatory
\end{abstract}

\section{Corresponding author:}

Assistant Professor Dr. Mandana khodashahi, Assistant Professor, Rheumatology Department, Rheumatic Diseases Research Center, Qaem Hospital, Mashhad University of Medical Sciences (MUMS), Mashhad, Iran.

Tel: +985138012753, Fax: +985138410136, Email: khodashahim921@mums.ac.ir

Received: October 22, 2015, Accepted: April 19, 2016, Published: August 2016

iThenticate screening: April 19, 2016, English editing: July 14, 2016, Quality control: August 04, 2016

(C) 2016 The Authors. This is an open access article under the terms of the Creative Commons Attribution-NonCommercialNoDerivs License, which permits use and distribution in any medium, provided the original work is properly cited, the use is non-commercial and no modifications or adaptations are made. 


\section{Introduction}

Rheumatoid arthritis (RA) is a chronic synovial inflammatory disorder with an unknown etiology, occurring in approximately $1 \%$ of the general population. The incidence of RA in females is 2.5 times higher than males, and its prevalence increases with age in both men and women $(1,2)$. Early diagnosis and prompt treatment can prevent joint destruction and organ damage in this disease $(3,4)$. The main objectives of RA treatment include pain relief, inflammation reduction, joint function preservation, prevention of systemic involvement, and reducing morbidity. Non-biologic Disease-Modifying Anti-Rheumatic Drugs (DMARDs), biologic DMARD agents, glucocorticoids, and rehabilitation are known to be effective RA treatments and play an essential role in the management of symptoms and disease progression (5). Atorvastatin, as a lipid-lowering agent, prevents the production of cholesterol in the liver by inhibiting HMG-CoA reductase. This agent reduces total cholesterol and low-density lipoprotein (LDL) levels $(6,7)$. Pro-inflammatory cytokines, including IL-6, IL-8, IL-1B, and TNF $\alpha$, as well as chemokines such as CCL5, CCL2, MMP9, and NF-KB, significantly decrease during treatment with this agent. Moreover, interferon-gamma and cytokine concentrations significantly decrease in the T-cell culture of RA patients (8). However, the upregulation of CD59, decay-accelerating factor in hypoxia, and IL-10 levels increase with atrovastatin treatment (8-10). Overall, atrovastatin improves the endothelial function through elasticity enhancement and decreases atheromatous plaque formation, endothelial damage, and systemic inflammation $(6,11,12)$. This study aimed to determine the anti-inflammatory effect of atorvastatin on the Disease Activity Index and high-density lipoprotein (HDL) in RA patients, with regards to different genetic and epidemiologic factors associated with RA in the Iranian population.

\section{Material and Methods}

\subsection{Trail design}

This double-blind, randomized clinical trial was conducted from November 2013 to November 2014 in academic hospitals affiliated with the Mashhad University of Medical Sciences in Mashhad, Iran.

\subsection{Sample size}

The optimal sample size was calculated to be 38 subjects. This sample size was estimated based on the mean hsCRP reported in previous studies (5) with $\mathrm{X}_{2}=10.4, \mathrm{~S}_{2}=9.4, \mathrm{X}_{1}=3.2, \mathrm{~S}_{1}=3.5, \alpha=105, \beta=12$. Assumptions include a test power of $80 \%$ and a confidence level of $95 \%$ and the use of the following formula: $\mathrm{n}=\left(\mathrm{Z}_{1}-\alpha / 2+\mathrm{Z}_{1}-\beta\right)^{2} \times\left(\left(\mathrm{P}_{1} \times\left(1-\mathrm{P}_{1}\right)+\mathrm{P}_{2} \times\left(1-\mathrm{P}_{2}\right)\right) /\left(\mathrm{P}_{1}-\mathrm{P}_{2}\right)^{2}\right.$

\subsection{Participants}

Thirty-eight RA patients, diagnosed by the 2010 American College of Rheumatology (ACR)/European League against Rheumatism (EULAR) criteria, were recruited in 2013-2014 from Imam Reza and Ghaem hospitals, which are affiliated to Mashhad University of Medical Sciences (MUMS).

\subsection{Selection criteria}

The inclusion criteria were as follows: 1) meeting the 2010 ACR/EULAR criteria; and 2) $\geqslant 18$ years of age. The exclusion criteria were as follows: 1) known liver disease; 2) elevated transaminase levels two months before the study; 3) previous history of myopathy; 4) pregnancy; 5) breastfeeding; 6) use of lipid-lowering agents; and 7) being eligible for taking a lipid-lowering agents according to the National Cholesterol Education Program (NCEP).

\subsection{Randomization and blinding}

Patients were randomly divided into two groups using the random block method. Our rheumatologists were blinded to treatment intervention during study.

\subsection{Interventions and data collection}

All data were collected using a checklist to record the observational results. The participants were randomly divided into two groups: 1) the intervention group taking atorvastatin $40 \mathrm{mg}$ daily with DMARDs (19 subjects), and 2) the control group receiving DMARDs without atorvastatin (19 subjects). The patients received hydroxychloroquine (400 mg/day), methotrexate (15- $25 \mathrm{mg} /$ weekly), sulfasalazine (1-2 gr/day), and prednisolone (5-10 mg) on a daily basis for 12 weeks. Atorvastatin ( $40 \mathrm{mg}$ daily) was added to the regimen of the intervention group. All patients were evaluated at weeks zero, four, eight, and twelve by two rheumatologists. Response to treatment and the clinical status of patients were evaluated using the Disease Activity Score (DAS-28) and Visual Analogue Scale (VAS) at weeks zero, four, eight, and twelve, based on the 2010 ACR/EULAR criteria (1) by two rheumatologists. Disease activity and laboratory parameters, including erythrocyte sedimentation rate (ESR), high-sensitivity C-reactive 
protein (hs-CRP), DAS-ESR, high-density lipoprotein (HDL), LDL, and liver function test, were measured in both study groups. During each visit, the clinical status of the patients was evaluated according to DAS28-ESR and VAS criteria. Also, blood samples were obtained to determine ESR, hs-CRP, liver function test (LFT), HDL, LDL, and total cholesterol. Additionally, response to treatment was assessed by the EULAR criteria (1).

\subsection{Statistical methods}

All data were entered to SPSS version 13.1. Friedman test, repeated measurement method, t-test, Chi-square, and ANOVA were used for data analysis. P-values less than 0.05 were considered statistically significant.

\subsection{Research ethics}

Informed consents were obtained from all patients and the study was approved by the Ethic Committee of MUMS (IR.mums.REC.1389.71).

\section{Results}

From 38 enrolled patients enrolled the study, 19 patients were assigned to the intervention group and 19 patients to the control group. Demographic data showed that $89.8 \%$ and $99.1 \%$ of patients were female in the control and intervention groups, respectively. Figure 1 shows the CONSORT flow diagram of the trial. The mean age of the patients was $48.8 \pm 12.3$ years in the control group and $43.1 \pm 21.4$ years in the intervention group. The mean duration of the disease was $8.7 \pm 7.8$ and $6.6 \pm 5.6$ years in the control and intervention groups, respectively. Demographic characteristics showed no significant difference between the mean age of patients $(p=0.16)$ and disease duration $(\mathrm{p}=0.36)$ between the groups (Table 1$)$.

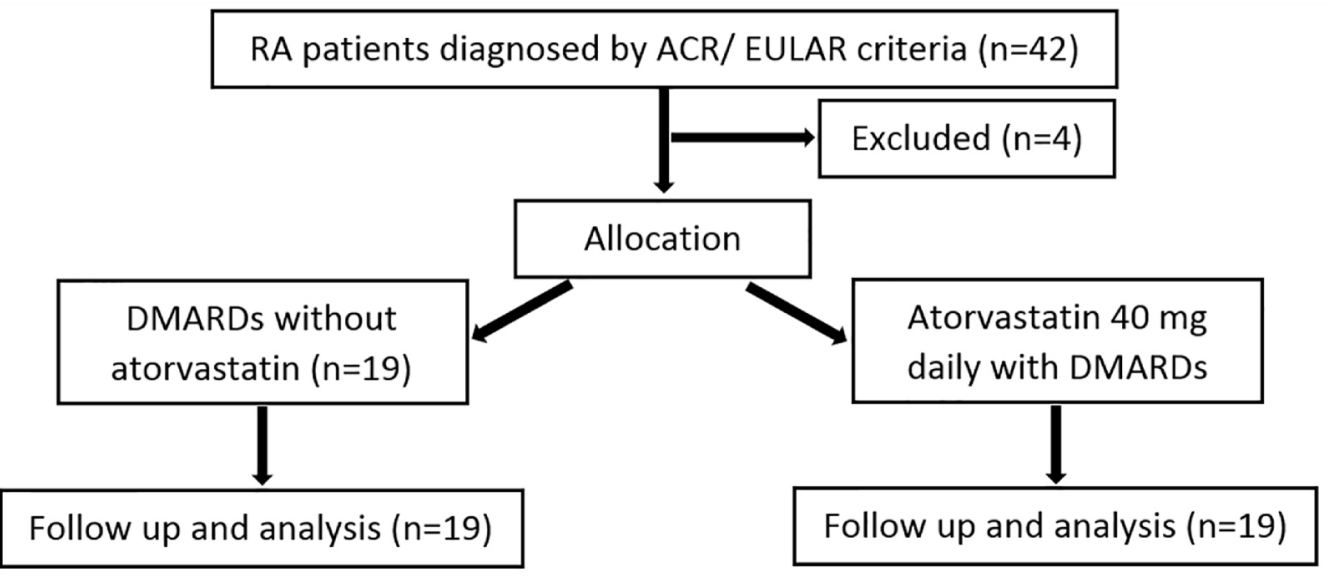

Figure 1. CONSORT flow diagram of the trial

Table 1. Base line data and demographic characteristics

\begin{tabular}{|l|l|l|l|}
\hline Characteristics & Control $(\mathrm{n}=19)$ & Case $(\mathrm{n}=19)$ & $\mathrm{p}$-value \\
\hline Age (yrs) & $48.8 \pm 12.3$ & $43.1 \pm 12.4$ & 0.163 \\
\hline Disease Duration (yrs) & $8.7 \pm 7.8$ & $6.5 \pm 5.6$ & 0.362 \\
\hline Patient's global assessment of disease activity (VAS) & $51.5 \pm 25.6$ & $51.8 \pm 24.2$ & 0.715 \\
\hline Swelling (n) & $3.1 \pm 2.9$ & $2.3 \pm 1.4$ & 0.011 \\
\hline Tenderness (n) & $7.5 \pm 5.2$ & $9.2 \pm 4.3$ & 0.385 \\
\hline Cholesterol (mg/dl) & $213.5 \pm 62.8$ & $171 \pm 30.5$ & 0.285 \\
\hline Triglyceride(mg/dl) & $151.4 \pm 85.1$ & $114.3 \pm 44.6$ & 0.331 \\
\hline HDL (mg/dl) & $53.3 \pm 8.3$ & $53.7 \pm 8.5$ & 0.016 \\
\hline LDL (mg/dl) & $134.5 \pm 22.6$ & $112.3 \pm 20.3$ & 0.036 \\
\hline SGOT & $32.2 \pm 9.1$ & $33.8 \pm 17.9$ & 0.185 \\
\hline SGPT & $15.8 \pm 5.0$ & $13.7 \pm 4.0$ & 0.122 \\
\hline ALKP & $135.5 \pm 51.5$ & $108.4 \pm 46.0$ & 0.429 \\
\hline ESR & $39.6 \pm 9.1$ & $34.4 \pm 9.6$ & 0.006 \\
\hline CRP & $16.4 \pm 15.7$ & $8.5 \pm 10.1$ & 0.628 \\
\hline DAS28_ESR & $5.1 \pm 0.85$ & $4.7 \pm 0.87$ & 0.043 \\
\hline
\end{tabular}


According to clinical findings, the mean number of tender and swollen joints was $7.5 \pm 5.2$ and $3.1 \pm 2.9$ in the control group and $9.2 \pm 4.3$ and $2.3 \pm 1.4$ in the intervention group, respectively (Table 2). Based on VAS, the mean self-assessment score of disease activity was $51.6 \pm 25.7$ in the control group and $51.8 \pm 24.2$ in the intervention group. The mean values of ESR, hs-CRP, DAS28-ESR, and DAS28-hs-CRP were 39.6 $\pm 9.1,16.5 \pm 15.8,5.1 \pm$ 0.85 , and $4.5 \pm 7.8$ in the control group, respectively (Table 1). Also, these values were $34.4 \pm 9.6,8.5 \pm 10.1,4.8 \pm$ 0.88 , and $4.7 \pm 0.87$ in the intervention group, respectively (Table 2, Figure 2).

Table 2. Data on swelling joints during the study

\begin{tabular}{|c|c|c|c|}
\hline Time & Control $(n=19)$ & Case $(n=19)$ & p-value* \\
\hline Swelling (week 0) & $3.1 \pm 2.9$ & $2.3 \pm 1.4$ & \multirow[t]{4}{*}{0.011} \\
\hline Swelling (week $4^{\text {th }}$ ) & $3.0 \pm 3.3$ & $0.89 \pm 1.1$ & \\
\hline Swelling (week $8^{\text {th }}$ ) & $2.4 \pm 2.9$ & $1.1 \pm 1.3$ & \\
\hline Swelling (week $12^{\text {th }}$ ) & $2.6 \pm 3.4$ & $0.57 \pm 0.83$ & \\
\hline
\end{tabular}

*According to Repeated Measures method for analysis and Hotelling's Trace test.
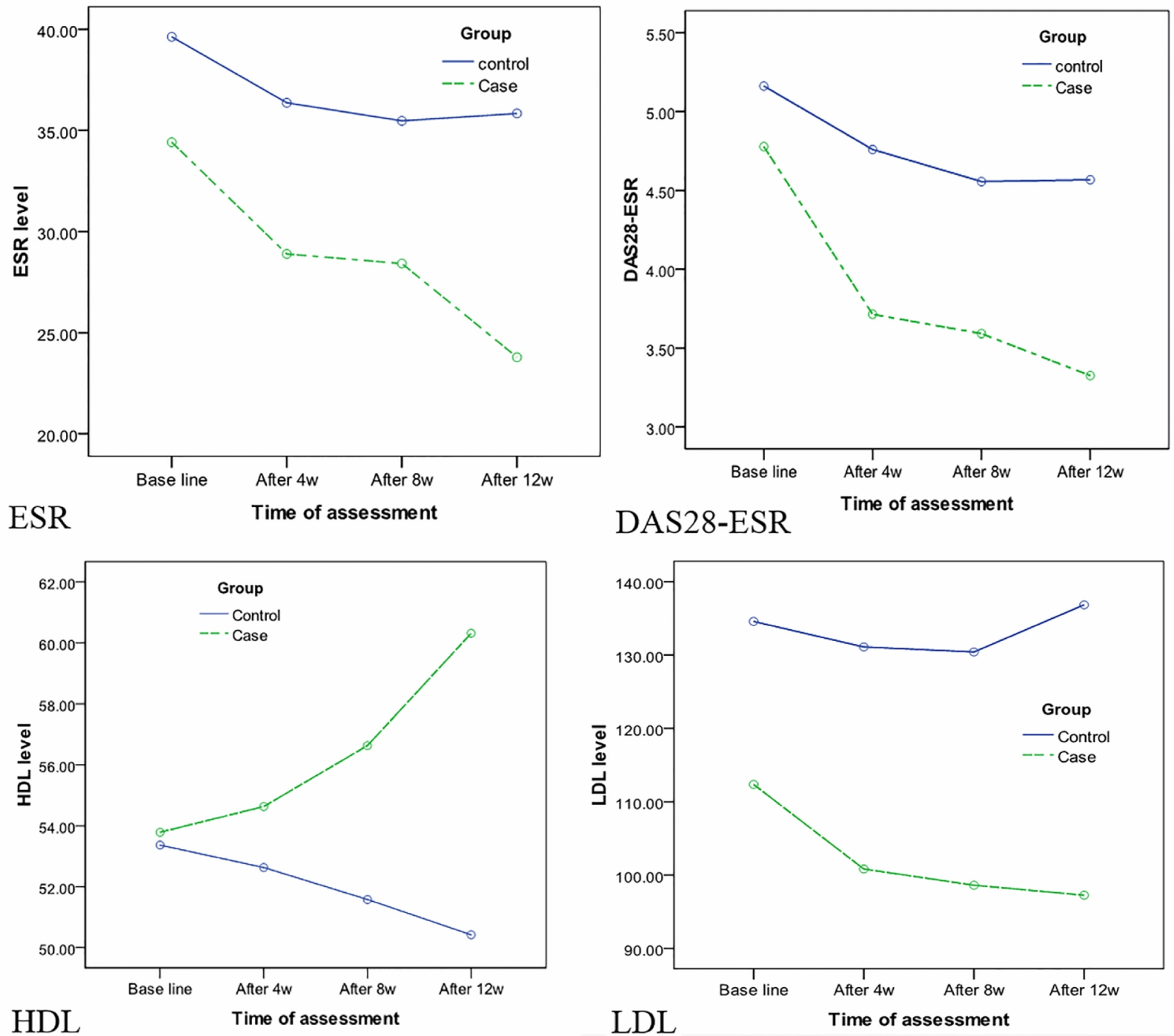

Figure 2. Laboratory findings.

Statistical analysis showed a significant difference in ESR $(\mathrm{p}=0.006)$ between the intervention and control groups. However, there was no significant difference between the two groups in terms of joint tenderness $(\mathrm{p}=0.28)$ or hsCRP ( $\mathrm{p}=0.07$ ) (Figure 2). The laboratory tests related to lipid profile showed that the mean values of LDL, HDL, 
$\mathrm{TG}$, and total cholesterol were $134.5 \pm 22.6,53.36 \pm 8.3,151.4 \pm 85.2$, and $213.5 \pm 62.8$ in the control group, respectively. In the intervention group, the corresponding values were $112.3 \pm 20.8,53.7 \pm 8.5,114.4 \pm 44.7$, and $171.0 \pm 30.6$, respectively. There was a significant difference in LDL $(p=0.036)$ and HDL $(p=0.016)$ between the two groups. However, no significant differences were reported between the two groups in terms of cholesterol $(\mathrm{p}=0.28)$ or $\mathrm{TG}(\mathrm{p}=0.33)$. The findings of LFT showed that the mean values of serum glutamic oxaloacetic transaminase (SGOT), serum glutamic pyruvate transaminase (SGPT), and alkaline phosphatase (ALP) were $32.2 \pm$ 9.2, $15.8 \pm 5.0$, and 135.6 \pm 51.6 in the control group, respectively. Also, the corresponding values were $33.8 \pm 17.9$, $13.7 \pm 4.0$, and $108.5 \pm 46.0$ in the intervention group, respectively. Liver function tests showed no significant difference in SGOT $(p=0.18)$, SGPT $(p=0.12)$, or ALP $(p=0.42)$ between the two groups. There was a significant difference in the number of swollen joints $(p=0.011)$, ESR $(p=0.006)$, and DAS-ESR $(p=0.043)$ between the groups. However, no significant difference was observed between the two groups in terms of tender joints $(\mathrm{p}=0.38)$, VAS score ( $\mathrm{p}=0.71)$, hs-CRP ( $\mathrm{p}=0.62)$, or DAS28-hs-CRP ( $\mathrm{p}=0.431)$ according to Hotelling's Trace coefficient test. Nevertheless, there was no significant difference in total cholesterol $(\mathrm{p}=0.28)$ or $\mathrm{TG}(\mathrm{p}=0.33)$ (Figure 2). A comparison between the control and intervention groups showed no significant difference in SGOT ( $\mathrm{p}=0.18)$, SGPT $(\mathrm{p}=0.12)$, or ALP $(\mathrm{p}=0.42)$. At the end of week 12 , according to the EULAR criteria, $38.5 \%$ of patients in the control group and $55.6 \%$ of patients in the intervention group showed moderate or good responses to treatments. In total, $48.4 \%$ of patients in the intervention and control groups showed moderate or good responses. However, according to the Chi-square test, there was no significant difference in response to treatment between the two groups.

\section{Discussion}

This study revealed a significant improvement in the number of swollen joints. The number of swollen joints in the intervention group was significantly lower than that observed in the control group. However, the number of tender joints was not significantly different between the two groups. In general, statins have an impact on innate immune responses through activation of endothelial cells (13), macrophages, neutrophils, and natural killer cells (14). In addition, these agents have similar effects on acquired immune responses through suppression of antigen-presenting cells and polarized T-cells (15). The inflammation of synovial membranes in RA arises with the activities of both the innate and acquired immune systems. Therefore, atorvastatin may contribute to the improvement of inflammation and, consequently joint swelling, as confirmed by previous studies $(4,13,16,17)$. According to the obtained results, the simultaneous use of atorvastatin $(40 \mathrm{mg} /$ day) and DMARDs leads to a significant reduction in ESR. This finding was consistent with studies performed by McCarey-McInnes et al. in 2004 and Kinlay et al. in $2003(13,10)$. DAS28-ESR assessments demonstrated a statistically significant difference between the two groups. In addition, lipid profiles decreased during the intervention, which was consistent with the results obtained by Charles-Schoeman et al. in 2007 and McCarey et al. in $2004(5,13)$. Moreover, previously conducted studies revealed that atorvastatin does not reduce HDL or TG level $(18,19,20)$. These results differ from our findings, which demonstrated a significant reduction in serum HDL and LDL levels. As shown by some previous studies, the side-effects of atorvastatin are not dose-dependent (21). According to a study by Athyros et al., the most common and serious side-effects of atorvastatin are hepatotoxicity and myopathy, which was similar to the results of our study $(15,22)$. The main limitation of our study was small sample size. Therefore, authors propose to conduct the study in a larger population.

\section{Conclusions}

This study showed the positive effect of atorvastatin on the disease course of RA. Although DMARD is the first-line treatment for RA, atorvastatin may also improve RA inflammation. In our study, the number of swollen joints in the atorvastatin group decreased more significantly than the control group. Since RA is a chronic, debilitating, autoimmune disorder that causes irreversible joint damage, all patients need to be treated promptly and effectively with different combinations of traditional DMARDs in order to minimize joint destruction (9). Further studies are required to determine the most effective therapy, which may include addition of atorvastatin to conventional DMARDs before initiating biologic DMARDs.

\section{Acknowledgments:}

The researchers would like to thank the staff and nurses of Qaem and Emam Reza hospitals, without whom conducting this study would not have been possible. This research project was financially supported by the Rheumatic Diseases Research center at the Mashhad University of Medical Sciences. It was also the subspecialty thesis of Mehran Ghazi Saeidi. 


\section{Trial registration:}

The trial was registered at the Iranian Registry of Clinical Trials (http://www.irct.ir) with the Irct ID: IRCT911259162N1.

\section{Funding:}

The authors received no financial support for the research, authorship, and/or publication of this article.

\section{Conflict of Interest:}

There is no conflict of interest to be declared.

\section{Authors' contributions:}

All authors contributed to this project and article equally. All authors read and approved the final manuscript.

\section{References:}

1) Aletaha D, Neogi T, Silman AJ, Funovits J, Felson DT, Bingham CO, et al. 2010 rheumatoid arthritis classification criteria: an American College of Rheumatology/European League Against Rheumatism collaborative initiative. Arthritis Rheum. 2010; 62(9): 2569-81. doi: 10.1002/art.27584. PMID: 20872595.

2) Athyros VG, Papageorgiou AA, Athyrou VV, Demitriadis DS, Kontopoulos AG. Atorvastatin and micronized fenofibrate alone and in combination in type 2 diabetes with combined hyperlipidemia. Diabetes Care. 2002; 25(7): 1198-202. doi: 10.2337/diacare.25.7.1198. PMID: 12087019.

3) Bansback N, Ara R, Ward S, Anis A, Choi HK. Statin Therapy in Rheumatoid Arthritis. Pharmacoeconomics. 2009; 27(1): 25-37. doi: 10.2165/00019053-200927010-00004. PMID: 19178122.

4) Barsante MM, Roffê E, Yokoro CM, Tafuri WL, Souza DG, Pinho V, et al. Anti-inflammatory and analgesic effects of atorvastatin in a rat model of adjuvant-induced arthritis. Eur J Pharmacol. 2005; 16(3): 282-9. doi: 10.1016/j.ejphar.2005.05.005. PMID: 15970284.

5) Charles-Schoeman C, Khanna D, Furst DE, McMahon M, Reddy ST, Fogelman AM, et al. Effects of highdose atorvastatin on antiinflammatory properties of high density lipoprotein in patients with rheumatoid arthritis: a pilot study. J Rheumatol. 2007; 34(7): 1459-64. PMID: 17552046.

6) Cush JJ, Weinblatt ME, Kavanaugh A. Rheumatoid Arthritis: Early Diagnosis and Treatment, 4E. Professional Communications. 2010.

7) Ebringer A. Rheumatoid Arthritis and Proteus. Springer. 2011.

8) Hermann M, Bogsrud MP, Molden E, Asberg A, Mohebi BU, Ose L, et al. Exposure of atorvastatin is unchanged but lactone and acid metabolites are increased several-fold in patients with atorvastatin-induced myopathy. Clin Pharmacol Ther. 2006; 79(6): 532-9. doi: 10.1016/j.clpt.2006.02.014. PMID: 16765141.

9) Katzung BG, Masters SB, Trevor AJ. Basic and Clinical Pharmacology 12/E. McGraw-Hill Education. 2012.

10) Kinlay S, Schwartz GG, Olsson AG, Rifai N, Leslie SJ, Sasiela WJ, et al. High-dose atorvastatin enhances the decline in inflammatory markers in patients with acute coronary syndromes in the MIRACL study. Circulation. 2003; 108(13): 1560-6. doi: 10.1161/01.CIR.0000091404.09558.AF. PMID: 12975259.

11) Longo DL, Fauci AS, Kasper DL, Hauser SL, Jameson JL, Loscalzo J. Harrison's Principles of Internal Medicine, 18th Edition. McGraw-Hill Education. 2011.

12) Macin SM, Perna ER, Farías EF, Franciosi V, Cialzeta JR, Brizuela M, et al. Atorvastatin has an important acute anti-inflammatory effect in patients with acute coronary syndrome: results of a randomized, doubleblind, placebo-controlled study. Am Heart J. 2005; 149(3): 451-7. doi: 10.1016/j.ahj.2004.07.041. PMID: 15864233.

13) McCarey DW, McInnes IB, Madhok R, Hampson R, Scherbakova O, Ford I, et al. Trial of Atorvastatin in Rheumatoid Arthritis (TARA): double-blind, randomised placebo-controlled trial. Lancet. 2004; 363(9426): 2015-21. doi: 10.1016/S0140-6736(04)16449-0. PMID: 15207950.

14) McInnes IB, Kim HY, Lee SH, Mandel D, Song YW, Connell CA, et al. Open-label tofacitinib and doubleblind atorvastatin in rheumatoid arthritis patients: a randomised study. Ann Rheum Dis. 2014; 73(1): 12431. doi: 10.1136/annrheumdis-2012-202442. PMID: 23482473.

15) Nikitina NM, Rebrov AP. [The use of atorvastatin in patients with rheumatoid arthritis with hyperlipidemia]. Kardiologiia. 2009; 49(9): 21-6. PMID: 19772499.

16) Susekov AV, Rozhkova TA, Tripoten MI, Pogorelova OA, Kulev BD, Balakhonova TV, et al. [Randomized FARVATER study. Part 2. Effect of atorvastatin on endothelial function, distensibility and stiffness of vascular wall]. Kardiologiia. 2006; 47(2): 25-30. PMID: 17495819. 
17) Wahane VD, Kumar VL. Atorvastatin ameliorates inflammatory hyperalgesia in rat model of monoarticular arthritis. Pharmacol Res. 2010; 61(4): 329-33. doi: 10.1016/j.phrs.2009.11.006. PMID: 19931618.

18) Yood RA. American College of Rheumatology Subcommittee on Rheumatoid Arthritis G. Guidelines for the management of rheumatoid arthritis: 2002 update. 2002.

19) Blaschke S, Viereck V, Schwarz G, Klinger HM, Guerluek S, Müller GA. Anti-inflammatory effects of atorvastatin on peripheral blood mononuclear cells and synovial fibroblasts in rheumatoid arthritis. Scand J Rheumatol. 2009; 38(4): 235-9. doi: 10.1080/03009740802572475. PMID: 19247848.

20) Kinderlerer AR, Steinberg R, Johns M, Harten SK, Lidington EA, Haskard DO, et al. Statin-induced expression of CD59 on vascular endothelium in hypoxia: a potential mechanism for the anti-inflammatory actions of statins in rheumatoid arthritis. Arthritis Res Ther. 2006; 8(4): 130. doi: 10.1186/ar2019. PMID: 16859540, PMCID: PMC1779384.

21) Barsante MM, Roffê E, Yokoro CM, Tafuri WL, Souza DG, Pinho V, et al. Anti-inflammatory and analgesic effects of atorvastatin in a rat model of adjuvant-induced arthritis. Eur J Pharmacol. 2005; 516(3): 282-9. doi: 10.1016/j.ejphar.2005.05.005. PMID: 15970284.

22) Tan KC, Chow WS, Tam SC, Ai VH, Lam CH, Lam KS. Atorvastatin lowers C-reactive protein and improves endothelium-dependent vasodilation in type 2 diabetes mellitus. J Clin Endocrinol Metab. 2002; 87(2): 563-8. doi: 10.1210/jcem.87.2.8249. PMID: 11836286. 